\title{
Heavy exotic quarkonia and doubly heavy baryons
}

\author{
Marek Karliner,a \\ ${ }^{1}$ Raymond and Beverly Sackler School of Physics and Astronomy \\ Tel Aviv University, Tel Aviv, Israel
}

\begin{abstract}
During the last three years strong experimental evidence from B and charm factories has been accumulating for the existence of exotic hadronic quarkonia, narrow resonances which cannot be made from a quark and an antiquark. Their masses and decay modes show that they contain a heavy quark-antiquark pair, but their quantum numbers are such that they must also contain a light quark-antiquark pair. The main theoretical challenge has been to determine the nature of these resonances. The main possibilities are that they are either "genuine tetraquarks", i.e. two quarks and two antiquarks within one confinement volume, or "hadronic molecules" of two heavy-light mesons. In the last few months there is more and more evidence in favor of the latter. In addition, there exist narrow resonances such as $X(3872)$ which are not exotic but are closely related to the exotic states. Most likely such states are a mixture of hadronic molecules and excited quarkonia. I discuss the experimental data and its interpretation and provide fairly precise predictions for masses and quantum numbers of the additional exotic states which are naturally expected in the molecular picture but have yet to be observed. In addition, I provide arguments in favor of the existence of an even more exotic state - a hypothetical deuteron-like bound state of two heavy baryons. I also consider "baryon-like" states $Q Q^{\prime} \bar{q} \bar{q}^{\prime}$, which if found will be direct evidence not just for near-threshold binding of two heavy mesons, but for genuine tetraquarks with novel color networks. I also stress the importance of experimental search for doubly-heavy baryons which are intimately connected with doubly heavy exotics.
\end{abstract}

\section{First observation of manifestly exotic hadrons}

In late 2007 the Belle Collaboration reported [1] anomalously large rate partial widths of $\Upsilon(5 S) \rightarrow \Upsilon(2 S)$ and $\Upsilon(5 S) \rightarrow \Upsilon(1 S)$, two orders of magnitude larger than the analogous decays of $\Upsilon(3 S)$. Soon afterward Harry Lipkin and I proposed [2] that a four-quark exotic resonance $[b \bar{b} u \bar{d}]$ might mediate these decays through the cascade $\Upsilon(m S) \rightarrow[b \bar{b} u \bar{d}] \pi^{-} \rightarrow \Upsilon(n S) \pi^{+} \pi^{-}$. We suggested looking for the $[b \bar{b} u \bar{d}]$ resonance in these decays as peaks in the invariant mass of $\Upsilon(1 S) \pi$ or $\Upsilon(2 S) \pi$ systems. More recently Belle collaboration confirmed this prediction, announcing $[3,4]$ the observation of two charged bottomonium-like resonances $Z_{b}$ as narrow structures in $\pi^{ \pm} \Upsilon(n S)(n=1,2,3)$ and $\pi^{ \pm} h_{b}(m P)(m=1,2)$ mass spectra that are produced in association with a single charged pion in $\Upsilon(5 S)$ decays. The measured masses of the two structures averaged over the five final states are $M_{1}=10608.4 \pm 2.0 \mathrm{MeV}, M_{2}=10653.2 \pm 1.5 \mathrm{MeV}$, both with a width of about $15 \mathrm{MeV}$. Interestingly enough, the two masses $M_{1}$ and $M_{2}$ are about $3 \mathrm{MeV}$ above the respective $B^{*} \bar{B}$ and $B^{*} \bar{B}^{*}$ thresholds.

This strongly suggests a parallel with $X(3872)$, whose mass is almost exactly at the $D^{*} \bar{D}$ threshold. It also raises the possibility that such states might have a complementary description as deuteron-like "molecule" of two heavy

\footnotetext{
a e-mail: marek@proton.tau.ac.il
}

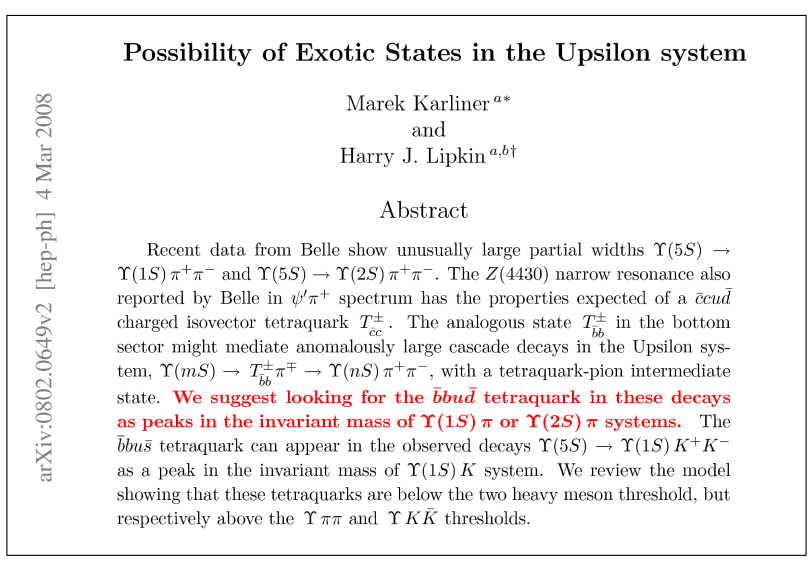

Figure 1. First page of our paper [2] proposing a four-quark exotic intermediate state $b \bar{b} u \bar{d}$ as an explanation of the anomalously large rate partial widths of $\Upsilon(5 S) \rightarrow \Upsilon(2 S)$ and $\Upsilon(5 S) \rightarrow \Upsilon(1 S)$.

mesons quasi-bound by pion exchange [6-8], as schematically shown in Figure 2

The attraction due to $\pi$ exchange is 3 times weaker in the $I=1$ channel than in the $I=0$ channel. Consequently, in the charm system the $I=1$ state is expected to be well above the $D^{*} \bar{D}$ threshold and the $I=0 \quad X(3872)$ is at the 

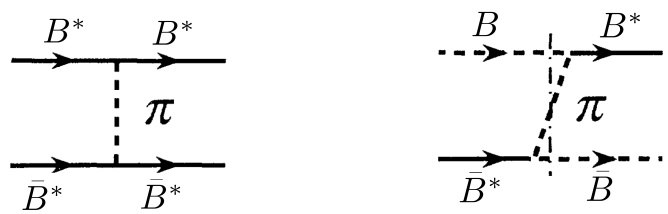

Figure 2. Diagrams contributing to $B \bar{B}^{*}$ and $B^{*} \bar{B}^{*}$ binding through pion exchange. Analogous diagrams contribute to $D \bar{D}^{*}$ and $D^{*} \bar{D}^{*}$ binding, modulo the caveat that $D^{*} \rightarrow D \pi$, while $B^{*} \rightarrow B \pi$ is kinematically forbidden, as discussed in the text.

threshold. ${ }^{1}$ In the bottom system the attraction due to $\pi$ exchange is essentially the same, but the kinetic energy is much smaller by a factor of $\sim m(B) / m(D) \approx 2.8$. Therefore the net binding is much stronger than in the charm system.

The recently discovered manifestly exotic charged resonances are surprisingly narrow. This is the case in both $\bar{b} b$ systems $[3,4]-$ and in the exotic charmonium, namely the remarkable peak $Z_{c}(3900)$ at $3899.0 \pm 3.6 \pm 4.9 \mathrm{MeV}$ with $\Gamma=46 \pm 10 \pm 20 \mathrm{MeV}$ reported by BESIII [5].

The relatively slow decay of these exotic resonances implies that the dominant configuration of the $\bar{Q} Q \bar{q} q$ fourbody system is not that of a low-lying $\bar{Q} Q$ quarkonium and pion(s). The latter have a much lower energy than the respective two-meson thresholds $\bar{M} M^{*}$ and $\bar{M}^{*} M^{*},(M=$ $D, B)$, but do readily fall apart into $(\bar{Q} Q)$ and pion(s) and would result in very large decay widths. We should view these systems as loosely bound states and/or near threshold resonances in the two heavy-meson system.

Such "molecular" states, $\bar{D} D^{*}$, etc., were introduced in Ref. [6]. They were later extensively discussed [7, 8] in analogy with the deuteron which binds via exchange of pions and other light mesons, and were referred to as "deusons". The key observation is that the coupling to the heavy mesons of the light mesons exchanged $(\pi, \rho$, etc.) becomes universal and independent of $M_{Q}$ for $M_{Q} \rightarrow \infty$, and so does the resulting potential in any given $J^{C P}$ and isospin channel. In this limit the kinetic energy $\sim p^{2} /\left(M_{Q}\right)$ vanishes, and the two heavy mesons bind with a binding energy $\sim$ the maximal depth of the attractive mesonexchange potential.

For a long time it was an important open question whether these consideration apply in the real world with large but finite masses of the $D$ and $B$ mesons. The recent experimental results of Belle [3, 4] and BESIII [5], together with theoretical analysis in Refs. [9] and [10] strongly indicate that such exotic states do exist - some were already found and more are predicted below.

Due to parity conservation, the pion cannot be exchanged in the $\bar{M} M$ system, but it does contribute in the $\bar{M} M^{*}$ and $\bar{M}^{*} M^{*}$ channels. The $\vec{\tau}_{1} \cdot \vec{\tau}_{s}$ isospin nature of the exchange implies that the the binding is 3 times stronger in the isoscalar channel. It was estimated $[9,10]$ that in the bottomonium system this difference in the binding potentials raises the $I=1$ exotics well above the $I=0$ exotics.

\footnotetext{
${ }^{1}$ For simplicity we treat $X(3872)$ as an isoscalar, since it has no charged partners, and we ignore here the issue of isospin breaking in its decays. A more refined treatment results in the same conclusions.
}

In the charmonium system this splitting is expected to be slightly smaller, because the $\bar{D} D^{*} / \bar{D}^{*} D^{*}$ states are larger than $\bar{B} B^{*} / \bar{B}^{*} B^{*}$. This is because the reduced mass in the $\bar{B} B^{*}$ system is approximately 2.5 times larger than in the $\bar{D} D^{*}$ system. On the other hand, the net attractive potential due to the light mesons exchanged between the heavy-light mesons is approximately the same, since $m_{c}, m_{b} \gg \Lambda_{Q C D}$. As usual in quantum mechanics, for a given potential the radius of a bound state or a resonance gets smaller when the reduced mass grows, so the $\bar{D} D^{*}$ states are larger than the $\bar{B} B^{*}$ states. Because of this difference in size the attraction in both $I=0$ and $I=1$ charmonium channels is expected to be somewhat smaller.

Since the quarks are heavy, we can treat their kinetic energy as a perturbation depending linearly on a parameter inversely proportional to $\mu_{\text {red }}$, the reduced mass of the two meson system, which scales like the mass of the heavy quark [11], with the hamiltonian $H=a \cdot p^{2}+V$, where $a=1 / \mu_{\text {red }} \sim 1 / m_{Q}$. We can use the existing data in order to make a very rough estimate of the isovector binding potential in the $m_{Q} \rightarrow \infty$ limit. We have two data points: $Z_{c}(3900)$ at $a(D)$ is approximately $27 \mathrm{MeV}$ above $\bar{D} D^{*}$ threshold and $Z_{b}(10610)$ at $a(B)$ is approximately 3 $\mathrm{MeV}$ above $\bar{B} B^{*}$ threshold. Linear extrapolation to $a=0$ yields $E_{b}^{I=1}(a=0) \approx-11.7 \mathrm{MeV}$. In view of the convexity, the actual binding energy is likely to slightly exceed this linear extrapolation.

We can then use this result for the isovector channel to estimate the $\bar{B} B^{*}$ binding in the isoscalar channel. Assuming that the isoscalar binding energy in the $m_{Q} \rightarrow \infty$ limit is 3 times larger than for the isovector, i.e. $E_{b}^{I=0}(a=0) \approx 3 \cdot(-11.7)=-35 \mathrm{MeV}$. $X(3872)$ is at $\bar{D} D^{*}$ threshold, providing an additional data point of $E_{b}^{I=0}(a(D)) \approx 0$ in the isoscalar channel. Linear extrapolation to $a(B)$ yields approximately $-20 \mathrm{MeV}$ as the $\bar{B} B^{*}$ binding energy in the isoscalar channel.

The upshot is that the newly discovered $Z_{c}(3900)$ isovector resonance confirms and refines the estimates in $[9,10]$ for the mass of the putative $\bar{B} B^{*}$ isoscalar bound state. This immediately lead to several predictions [11]:

a) two $I=0$ narrow resonances $X_{b}$ in the bottomonium system, about $23 \mathrm{MeV}$ below $Z_{b}(106010)$ and $Z_{b}(10650)$, i.e. about $20 \mathrm{MeV}$ below the corresponding $\bar{B} B^{*}$ and $\bar{B}^{*} B^{*}$ thresholds;

b) an $I=1$ resonance above $\overline{D^{*}} D^{*}$ threshold;

c) an $I=0$ resonance near $\overline{D^{*}} D^{*}$ threshold.

The $X_{b}$ states can most likely be observed through the decays $X_{b} \rightarrow \Upsilon \omega$ or $X_{b} \rightarrow \chi_{b} \pi \pi$. Unlike incorrectly stated in [11], they cannot decay to $\Upsilon \pi \pi$. The latter decays are prevented by $G$-parity conservation.[12] The observed decay $X(3872) \rightarrow J / \pi \pi$ is only possible because isospin is strongly broken between $D^{+}$and $D^{0}$, and because $X(3872)$ is at the $\bar{D} D^{*}$ threshold. In contradistinction, in the bottomonium system isospin is almost perfectly conserved. Thus the null result in CMS search [13] for $X_{b} \rightarrow \Upsilon(1 S) \pi^{+} \pi^{-}$does not tell us if $X_{b}$ exists.

Quite recently the BESIII collaboration reported observation in $e^{+} e^{-} \rightarrow\left(D^{*} \bar{D}^{*}\right)^{ \pm} \pi^{\mp}$ of what looks just like 


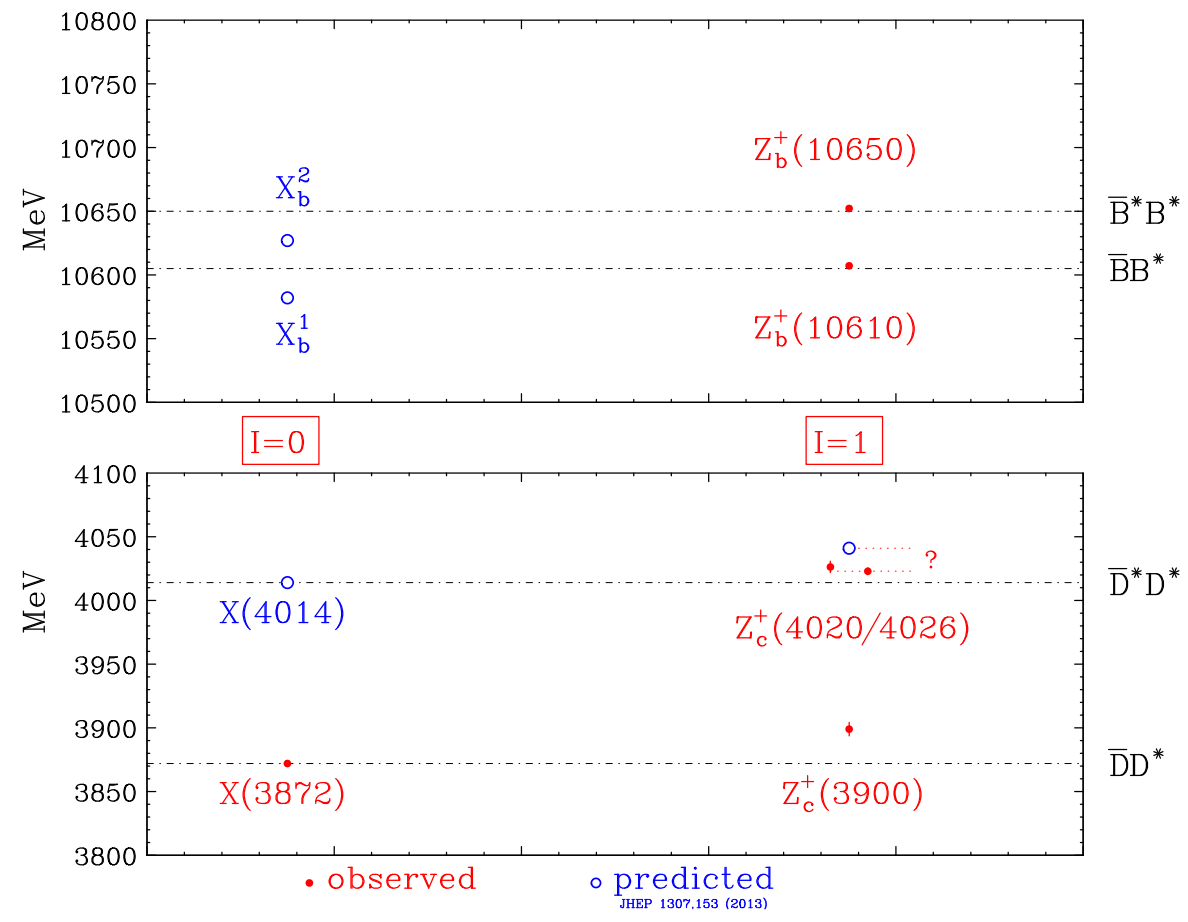

Figure 3. Masses of the doubly-heavy exotic quarkonia vs. two-meson thresholds. The states observed so far are shown in red, the predicted states are shown in blue. $I=0$ resonances are shown on the left and isovectors are shown on the right. Note the proximity of all the states to the corresponding two-meson thresholds.

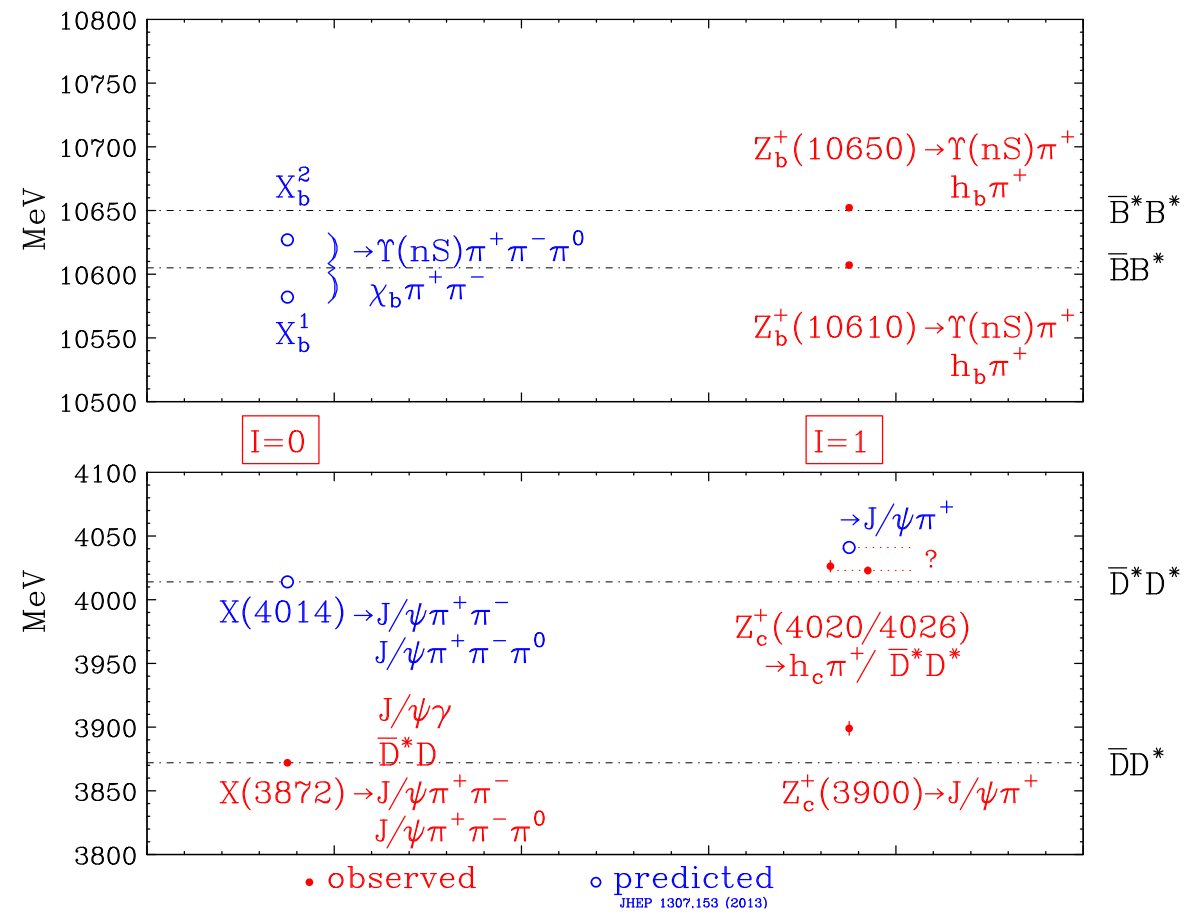

Figure 4. Decay channels of doubly-heavy exotic quarkonia. The legend is as in Figure 3. 
(b) above, namely a new charmonium-like charged resonance $Z_{c}(4025)$, slightly above the $\bar{D}^{*} D^{*}$ threshold, at $\sqrt{s}=(4026.3 \pm 2.6 \pm 3.7) \mathrm{MeV}$, with width of $24.8 \pm$ $5.6 \pm 7.7 \mathrm{MeV}$ [14]. Shortly afterward, BESIII reported observation of another charged charmoniumlike structure $Z_{c}$ (4020) in $e^{+} e^{-} \rightarrow \pi^{+} \pi^{-} h_{c}$ at $(4022.9 \pm 0.8 \pm 2.7) \mathrm{MeV}$ and width of $7.9 \pm 2.7 \pm 2.6 \mathrm{MeV}$. At this time it is not yet clear if these are two independent resonances, or two observations of the same object at slightly different masses, possibly due to systematic effects associated with the two observation channels.

Fig. 3 and 4 provide a concise summary of the experimental information about the masses of doubly-heavy exotics observed so far, together with our predictions for additional states, as discussed above.

It is somewhat puzzling that, unlike $Z_{c}^{+}(3900)$, $Z_{c}^{+}(4020 / 4026)$ has not been seen in the $J / \psi \pi^{+}$mode. Moreover, one notes that $Z_{c}^{+}(4020 / 4026)$ is somewhat closer to the $\bar{D}^{*} D^{*}$ threshold than our prediction. It will be interesting to identify the reasons for this small difference. The two main possibilities are (a) details of the experimental analysis; (b) a possible difference between the $\bar{B}^{*} B^{*}$ and $\bar{D}^{*} D^{*}$ attractive pion-exchange potentials. Such a difference might perhaps be due to the fact that $m\left(B^{*}\right)-[m(B)+$ $m(\pi)] \approx-94 \mathrm{MeV}$, while $m\left(D^{*}\right)-[m(D)+m(\pi)] \approx 0^{ \pm}$, depending on the $D^{*}$ and $\pi$ charges, affecting energy denominators in virtual pion emission.

\section{A $\left(\Sigma_{b}^{+} \Sigma_{b}^{-}\right)$beauteron dibaryon?}

The discovery of the $Z_{b}$-s and their interpretation as quasi-bound $B^{*} \bar{B}$ and $B^{*} \bar{B}^{*}$, raises an interesting possibility of a strongly bound $\Sigma_{b}^{+} \Sigma_{b}^{-}$deuteron-like state, a beauteron [16]. The $\Sigma_{b}$ is about $500 \mathrm{MeV}$ heavier than $B^{*}$. The $\Sigma_{b} \Sigma_{b}$ kinetic energy is therefore significantly smaller than that of $B \bar{B}^{*}$ or $B^{*} \bar{B}^{*}$. Moreover, $\Sigma_{b}$ with $I=1$ couples more strongly to pions than $B$ and $B^{*}$ with $I=\frac{1}{2}$. The opposite electric charges of $\Sigma_{b}^{+}$and $\Sigma_{b}^{-}$provide additional 2-3 MeV of binding energy. The heavy dibaryon bound state might be sufficiently long-lived to be observed experimentally. A possible decay mode of the beauteron is $\left(\Sigma_{b}^{+} \Sigma_{b}^{-}\right) \rightarrow \Lambda_{b} \Lambda_{b} \pi^{+} \pi^{-}$, which might be observable in LHCb. It should also be seen in lattice QCD.

\section{$2 Q Q \bar{q} \bar{q}$ tetraquarks}

The quark content of the exotic resonances observed so far is $\bar{Q} Q \bar{q} q$. A very different type of exotics are the $Q Q \bar{q}_{1} \bar{q}_{2}$ tetraquarks (TQ-s). [17-20] If such states do exist, producing and discovering even the lightest $c c \bar{u} \bar{d}$ is an extraordinary challenge. One needs to produce two $\bar{Q} Q$ pairs and then rearrange them, so as to form $Q Q$ and $\bar{Q} \bar{Q}$ diquarks, rather than the more favorable configuration of two $\bar{Q} Q$ and color singlets. Then the $Q Q$ diquark needs to pick up a $\bar{u} \bar{d}$ light diquark, rather than a $q$, to make a $Q Q q$ baryon, suppressing the production rate of these TQ-s below the rate of $Q Q q$ production.

As discussed below, a small ray of hope comes from the observation of the doubly-heavy $B_{c}=(\bar{b} c)$ mesons
[21], suggesting that simultaneous production of $\bar{b} b$ and $\bar{c} c$ pairs which are close to each other in space and in rapidity and can coalesce to form doubly-heavy hadrons is not too rare. As discussed in detail in the next section, this is an encouraging sign for the prospects of producing and observing the $c c q$ and $b c s$ baryons and hopefully also the $c c \bar{u} \bar{d}$ TQ. ATLAS and CMS and especially LHCb probably have the best chance of discovering these states. If the new TQ lies below say, the $D D^{*}$ threshold, it will be stable under the strong interaction and will decay only weakly.

Once the mass of the doubly heavy baryons is known, one can immediately estimate the mass of the corresponding tetraquark $[11,19]$ and check whether or not it is below the two $B$ meson threshold:

$m(b b \bar{u} \bar{d})=m\left(\Xi_{b b u}\right)+m\left(\Lambda_{b}\right)-m\left(B^{0}\right)-\frac{1}{4}\left[m\left(B^{*}\right)-m(B)\right]$

\section{Doubly-heavy baryons}

From the point of view of QCD there is nothing exotic about baryons containing two heavy quarks ( $b$ or $c$, generically denoted by $Q$ ) and one light quark ( $u$ or $d$, generically denoted by $q$ ). Heavy quarks decay only by weak interaction, with a characteristic lifetime orders of magnitude larger than the typical QCD timescale, so from the point of view of strong interactions the $Q Q q$ baryons are stable, just like protons, neutrons and hyperons. Thus these doubly-heavy baryons must exist.

On the other hand, producing and discovering them is an extraordinary challenge. One needs to produce two $\bar{Q} Q$ pairs and then rearrange them, so as to form $Q Q$ diquark. Then the $Q Q$ diquark needs to pick up a light quark $q$, to make a $Q Q q$ baryon. At first it seems that the production cross section for such a process is too low for the current generation of accelerators.

This view is probably overly pessimistic. A substantial basis for optimism is the observation of a large number of the doubly-heavy $B_{c}=(b \bar{c})$ mesons by D0, CDF and especially LHCb, [23-25] indicating [11, 22] that simultaneous production of $\bar{b} b$ and $\bar{c} c$ pairs which are close to each other in space and in rapidity and can coalesce to form doublyheavy hadrons is not too rare.

There are interesting parallels between the doublyheavy baryons $Q Q q$ and the hypothetical $Q Q \bar{q} \bar{q}$ tetraquarks. In both types of systems there is a light color triplet - a quark or an anti-diquark - bound to a heavy diquark. Because of this similarity, experimental observation of doubly-heavy baryons is very important not just in its own right, but as source of extremely valuable information for deducing the properties of the more exotic $Q Q \bar{q} \bar{q}$ tetraquarks. Such a deduction can carried out just as it was done for $b$-baryons.

In addition, doubly heavy baryons exhibit an interesting application of the heavy quark symmetry. In the limit $m_{Q} \rightarrow \infty$ the wave function of a light quark in a doubly heavy baryon $Q Q q$ is identical to the wave function of a light quark in a heavy light meson $\bar{Q} q$. This is because in 
a $Q Q q$ baryon the two heavy quarks have negligible kinetic energy and in the limit $m_{Q} \rightarrow \infty$ they become static, sitting on top of each other and forming a color antitriplet, just like in the $\bar{Q} q$ meson. Moreover, in the $m_{Q} \rightarrow \infty$ limit there are no spin-dependent interactions between the heavy and the light quarks, so it doesn't matter if the heavy color antritriplet is a fermion, like $\bar{Q}$ in the $\bar{Q} q$ meson or a boson, like the $Q Q$ diquark in the $Q Q q$ baryon.

Corrections to this baryon-meson relation scale like $\Lambda_{Q C D} / m_{Q}$ and are in principle computable. Thus the $Q Q q$ baryons will become a very useful "theoretical laboratory" for testing our ideas about bound state formation in QCD.

In the last few years it has become possible to accurately predict at the level of 2-3 MeV the masses of heavy baryons containing the $b$-quark: $\Sigma_{b}(b q q), \Xi_{b}(b s q)$ and $\Omega_{b}(b s s)$ [30-32], as shown in Figure 5. Similar approach can be used to predict the masses of doubly-heavy baryons [22], as shown in Table IV. Using these one can then compute the corresponding lifetimes, as shown in Table $\mathrm{V}$ which also shows other authors' estimates.

The most important decay modes are those involving the most-favored Cabibbo-Kobayashi-Maskawa matrix elements, such as $c \rightarrow s W^{*+}$ and $b \rightarrow c W^{*-}$. Among the latter, we focus on those modes which can pass the trigger criteria in the collider experiments, such as

(a) $\Xi_{c c}^{++}(c c u) \rightarrow(c s u) W^{*+} \rightarrow$

$$
\begin{aligned}
& \rightarrow \Xi_{c}^{+} \pi^{+} \rightarrow \\
& \rightarrow \Xi^{-} \pi^{+} \pi^{+} \pi^{+}, \Lambda_{c}^{+} K^{-} \pi^{+} \pi^{+}
\end{aligned}
$$

(b) $\Xi_{c c}^{+}(c c d) \rightarrow(c s d) W^{*+} \rightarrow$

$$
\rightarrow \Xi_{c}^{0} \pi^{+}, \Lambda_{c}^{+} K^{-} \pi^{+}
$$

(c) $\Xi_{b c}^{+}(b c u) \rightarrow \Xi_{c c}^{++} W^{*-}, \Xi_{b}^{0} W^{*+}$

(d) $\Xi_{b c}^{0}(b c d) \rightarrow \Xi_{c c}^{+} W^{*-}, \Xi_{b}^{-} W^{*+},(b s u)^{*}$

(e) $\Xi_{b b}(b b q) \rightarrow(b c q)^{*} W^{*-}$.

An interesting decay involving the subprocess $b \rightarrow$ $(\mathrm{J} / \psi s)$ twice is the chain

$$
\Xi_{b b} \rightarrow J / \psi \Xi_{b}^{(*)} \rightarrow J / \psi J / \psi \Xi^{(*)},
$$

where $\Xi_{b}^{(*)}$ denotes a (possibly excited) state with the minimum mass of $\Xi_{b}(5792)$, while $\Xi^{(*)}$ denotes a (possibly excited) state with the minimum mass of $\Xi$. Although this mode is expected to be quite rare and one has to pay the penalty of two $J / \psi$ leptonic branching fractions, it has a distinctive signature and is worth looking for.

In Ref. [22] we also estimated the hyperfine splitting between $B_{c}^{*}$ and $B_{c}$ mesons to be $68 \mathrm{MeV}$. P-wave excitations of the $\Xi_{c c}$ with light-quark total angular angular momentum $j=3 / 2$, the analog of those observed for $D$ and $B$ mesons, were estimated to lie around $420-470 \mathrm{MeV}$ above the spin-weighted average of the $\Xi_{c c}$ and $\Xi_{c c}^{*}$ masses.

\section{Prospects for detection}

Production of baryons containing two heavy quarks requires simultaneous production of two heavy quark-
Table IV. Summary of our mass predictions [22] (in $\mathrm{MeV}$ ) for lowest-lying baryons with two heavy quarks. States without a star have $J=1 / 2$; states with a star are their $J=3 / 2$ hyperfine partners. The quark $q$ can be either $u$ or $d$. The square or curved brackets around $c q$ denote coupling to spin 0 or 1 .

\begin{tabular}{cccc}
\hline \hline State & Quark content & $M(J=1 / 2)$ & $M(J=3 / 2)$ \\
\hline$\Xi_{c c}^{(*)}$ & $c c q$ & $3627 \pm 12$ & $3690 \pm 12$ \\
$\Xi_{b c}^{(*)}$ & $b[c q]$ & $6914 \pm 13$ & $6969 \pm 14$ \\
$\Xi_{b c}^{\prime}$ & $b(c q)$ & $6933 \pm 12$ & - \\
$\Xi_{b b}^{(*)}$ & $b b q$ & $10162 \pm 12$ & $10184 \pm 12$ \\
\hline \hline
\end{tabular}

antiquark pairs. Subsequently, a heavy quark from one pair needs to coalesce with a heavy quark from the other pair, forming together a color antitriplet heavy diquark. The heavy diquark then needs to pick up a light quark to finally hadronize as a doubly-heavy baryon. The coalescence of the two heavy quarks requires that they be in each other's vicinity in both ordinary space and in rapidity space. Computation of the corresponding cross section from first principles is difficult and is subject to considerable uncertainties due to nonperturbative effects. Instead, we use existing data [23-25] and theoretical estimates [33-35] of the closely-related process of $B_{c}$ production.

The two processes are closely related because production of $B_{c}$ also requires simultaneous production of two heavy quark-antiquark pairs. A priori, $B_{c}$ production has a somewhat higher probability, since in $B_{c}$ production a heavy quark from one pair needs to coalesce with a heavy antiquark (rather than a quark) from the other pair and there is no need to pick up an additional light quark. There is no suppression associated with the latter, as once the color anti-triplet heavy diquark is formed it can only hadronize by picking up a light quark. On the other hand, the attraction between a quark and an antiquark is two times stronger than the attraction between two quarks and we need to estimate the corresponding suppression factor. In order to see if $\Xi_{b c}$ and $B_{c}$ production rates are comparable, it would be useful to compare the analogous production rates of $\Xi_{c}$ and $D_{s}$ (or $\Xi_{b}$ and $B_{s}$ ) in experiments with large enough $E_{C M}$, whether in $e^{+} e^{-}, \bar{p} p$, or $p p$ collisions.

Although it is not directly related, one may consider the relative probability of a $b$ quark produced at high energy fragmenting into a meson (picking up a light antiquark) and a baryon (picking up a light diquark). The Heavy Flavor Averaging Group (HFAG) [36] has tabulated these quantities as measured in $\mathrm{Z}$ decays and the Tevatron.

According to the HFAG analysis, depending on the production mechanism, the $b$ quark turns into a baryon between about 10 and $25 \%$ of the time. Fragmentation into a baryon is somewhat favored at low transverse momentum [36] in hadron collisions.

More recently, LHCb has carried out a thorough analysis of the $b$ quark fragmentation into mesons and baryons [37-40]. In particular, the rather striking Fig. 4 in Ref. [40] shows that the ratio of $\Lambda_{b}$ production to $B^{0}$ meson produc- 


\section{b-baryons spectrum - TH predictions vs EXP}

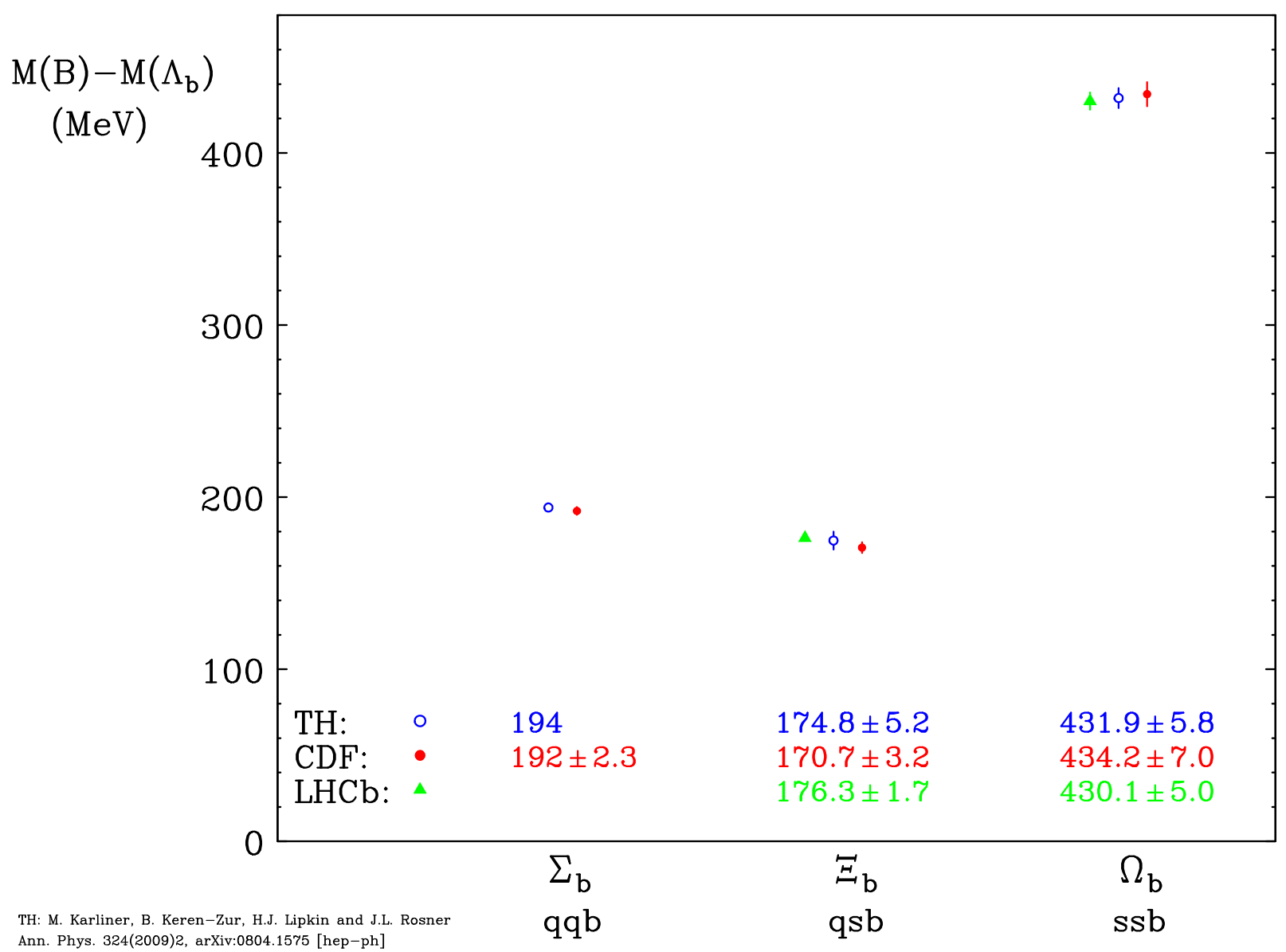

Figure 5. Masses of $b$-baryons - comparison of theoretical predictions[31, 32] with experiment.

Table V. Summary of lifetime predictions for baryons containing two heavy quarks. Values given are in fs.

\begin{tabular}{cccccc}
\hline \hline Baryon & Our work [22] & Ref. [26] & Ref. [27] & Ref. [28] & Ref. [29] \\
\hline$\Xi_{c c}^{++}=c c u$ & 185 & $430 \pm 100$ & $460 \pm 50$ & 500 & $\sim 200$ \\
$\Xi_{c c}^{+}=c c d$ & 53 & $120 \pm 100$ & $160 \pm 50$ & 150 & $\sim 100$ \\
$\Xi_{b c}^{+}=b c u$ & 244 & $330 \pm 80$ & $300 \pm 30$ & 200 & - \\
$\Xi_{b c}^{0}=b c d$ & 93 & $280 \pm 70$ & $270 \pm 30$ & 150 & - \\
$\Xi_{b b}^{0}=b b u$ & 370 & - & $790 \pm 20$ & - & - \\
$\Xi_{b b}^{-}=b b d$ & 370 & - & $800 \pm 20$ & - & - \\
\hline \hline
\end{tabular}

tion for $p_{T}$ below $10 \mathrm{GeV}$ is above 0.3 and goes above 0.5 for lower $p_{T}$.

A crude conclusion which we might draw from this comparison is that a baryon composed of two heavy quarks could be produced with at least as $10 \%$ of the $B_{c}$ production rate. An even more optimistic estimate, supported by the above LHCb fragmentation data, is provided by an explicit calculation [26] which predicts the production rates for $\Xi_{c c}$ and $\Xi_{b c}$ to be as large as $50 \%$ of that for $\left(B_{c}+B_{c}^{*}\right)$ at the Tevatron, of the order of several nb. The cross section for $\Xi_{b b}$ is estimated in that work to be about a factor of 10 less.
In [22] we computed the inclusive $B_{c}$ production cross section at the LHC directly from the $\mathrm{LHCb}$ data, obtaining

$$
\sigma\left(p p \rightarrow B_{c}+X\right) \approx 0.4 \mu \mathrm{b}
$$

for $4<p_{T}<40 \mathrm{GeV}$ and $2.5<\eta<4.5$. With $162 \pm 18$ $B_{c}^{+} \rightarrow J / 4 \pi^{+}$events we estimated [22] an acceptance a bit below $3 \%$. One might expect the $\Xi_{c c}$ production cross section at $\mathrm{LHCb}$ to be at most a tenth of $B_{c}$ cross section, i.e. $\sim 40 \mathrm{nb}$, at $7 \mathrm{TeV}$.

There is an interesting question whether $\Xi_{c c}$ is LHCb's best bet for discovering doubly-heavy baryons. The point is that because of Cabibbo suppression the $b$ quark lifetime is about 7 times longer than the $c$ quark, even though the $b$ quark is more than 3 times heavier and the phase space for 
weak quark decay of a heavy quark scales like $\left(m_{b} / m_{c}\right)^{5}$ times a kinematic function of the final and initial masses. Thus $\tau\left(\Lambda_{b}\right) \approx 1.5 \times 10^{-12}$ s vs. $\tau\left(\Lambda_{c}\right) \approx 2 \times 10^{-13} \mathrm{~s}$, etc. The difference between actual $\Xi_{c c}$ and $\Xi_{b c}$ lifetimes, as shown in Table 3, is not so pronounced. Longer lifetime makes it much easier to identify the secondary vertex. On the other hand, the cross section for producing bottom quarks is of course much smaller than for charmed quarks. So there is a tradeoff.

For sake of completeness, we also provide here a brief update on the status of search for doubly charmed baryons in $e^{+} e^{-}$experiments. The most recent and most stringent limits in this case come from Belle [41]. They used a 980 $\mathrm{fb}^{-1}$ data sample to search for $\Xi_{c c}^{+}$and $\Xi_{c c}^{++}$decaying into $\Lambda_{c}^{+} K^{-} \pi^{+}\left(\pi^{+}\right)$and $\Xi_{c}^{0} \pi^{+}\left(\pi^{+}\right)$final states.

Theoretical predictions for the inclusive cross section $\sigma\left(e^{+} e^{-} \rightarrow \Xi_{c c}+X\right)$ at Belle CM energy, $\sqrt{s}=10.58 \mathrm{GeV}$, vary over a rather wide range, from $70 \mathrm{fb}$ [42] to $230 \mathrm{fb}$ [43].

The CM energy of the B factories is sufficient only for production of $\Xi_{c c}$, as $\Xi_{b c}$ and $\Xi_{b b}$ are too heavy. So within the foreseeable future the latter can only be produced at LHC and perhaps at RHIC.

As in the case of doubly-heavy baryon production in $\mathrm{LHCb}$, there is a significant uncertainty in theoretical predictions for the inclusive cross section $\sigma\left(e^{+} e^{-} \rightarrow \Xi_{c c}+X\right)$. Therefore, we suggest another approach, similar in spirit to what we proposed for $\mathrm{LHCb}$. This approach is again directly based on observables which are in principle accessible in $e^{+} e^{-}$machines.

One can make a rough estimate of the doubly-charmed baryon production rate by assuming that the suppression of $c c q$ baryons $\Xi_{c c}$ vs. $c s q$ baryons $\Xi_{c}$ is of the same order of magnitude as the suppression of $\Xi_{c}$ vs. $s s q$ baryons $\Xi$ [22]. The physical content of this assumption is that the suppression due to replacing an $s$ quark in a baryon by a much heavier $c$ quark is approximately independent of the spectator quarks in the baryon:

$$
\begin{aligned}
& \sigma\left(e^{+} e^{-} \rightarrow \Xi_{c c}+X\right) \sim \\
\sim & \sigma\left(e^{+} e^{-} \rightarrow \Xi_{c}+X\right) \cdot \frac{\sigma\left(e^{+} e^{-} \rightarrow \Xi_{c}+X\right)}{\sigma\left(e^{+} e^{-} \rightarrow \Xi+X\right)}
\end{aligned}
$$

The approximate formula in Eq. (4) and its generalizations to $\Xi_{b c}$ and $\Xi_{b b}$ production should also apply to $p p$ collisions:

$$
\begin{aligned}
\sigma\left(p p \rightarrow \Xi_{b c}+X\right) & \sim \sigma\left(p p \rightarrow \Xi_{b}+X\right) \cdot \frac{\sigma\left(p p \rightarrow \Xi_{c}+X\right)}{\sigma(p p \rightarrow \Xi+X)} \\
& \sim \sigma\left(p p \rightarrow \Xi_{c}+X\right) \cdot \frac{\sigma\left(p p \rightarrow \Xi_{b}+X\right)}{\sigma(p p \rightarrow \Xi+X)}
\end{aligned}
$$

as well as

$$
\sigma\left(p p \rightarrow \Xi_{b b}+X\right) \sim \sigma\left(p p \rightarrow \Xi_{b}+X\right) \cdot \frac{\sigma\left(p p \rightarrow \Xi_{b}+X\right)}{\sigma(p p \rightarrow \Xi+X)} .
$$

We are optimistic that with the increased data samples soon to be available in hadronic and $e^{+} e^{-}$collisions, the first baryons with two heavy quarks will finally be seen.

\section{Acknowledgments}

The work described here was done in collaboration with B. Keren-Zur, H.J. Lipkin, J. Rosner and N. A. Tornqvist. It was supported in part by a grant from the Israel Science Foundation. The work of J. Rosner was supported by the U.S. Department of Energy, Division of High Energy Physics, Grant No. DE-FG02-13ER41958.

\section{References}

[1] K. F. Chen et al. [Belle Collaboration], Phys. Rev. Lett. 100, 112001 (2008) [arXiv:0710.2577 [hep-ex]].

[2] M. Karliner and H. J. Lipkin, arXiv:0802.0649 [hep$\mathrm{ph}]$.

[3] I. Adachi et al., Belle Coll., arXiv:1105.4583 [hepex].

[4] A. Bondar et al. [Belle Collaboration], Phys. Rev. Lett. 108, 122001 (2012) [arXiv:1110.2251 [hep-ex]].

[5] M. Ablikim et al. [ BESIII Collaboration], arXiv:1303.5949 [hep-ex].

[6] M. B. Voloshin and L. B. Okun, JETP Lett. 23, 333 (1976) [Pisma Zh. Eksp. Teor. Fiz. 23, 369 (1976)].

[7] N. A. Tornqvist, Phys. Rev. Lett. 67, 556 (1991); N. A. Tornqvist, Z. Phys. C 61, 525 (1994) [hep$\mathrm{ph} / 9310247]$.

[8] N. A. Tornqvist, Phys. Lett. B 590, 209 (2004) [hep$\mathrm{ph} / 0402237]$.

[9] M. Karliner, H. J. Lipkin, N. A. Tornqvist, arXiv:1109.3472 [hep-ph].

[10] M. Karliner, H. J. Lipkin, N. A. Tornqvist, Nucl. Phys. Proc. Suppl. 225-227, 102 (2012).

[11] M. Karliner and S. Nussinov, JHEP 1307, 153 (2013) [arXiv:1304.0345 [hep-ph]].

[12] I thank Roman Mizuk for discussion on this point.

[13] S. Chatrchyan et al. [CMS Collaboration], arXiv:1309.0250 [hep-ex].

[14] M. Ablikim et al. [BESIII Collaboration], arXiv:1308.2760 [hep-ex].

[15] M. Ablikim et al. [BESIII Collaboration], arXiv:1309.1896 [hep-ex].

[16] M. Karliner, H.J. Lipkin, N.A. Törnqvist, unpublished and [arXiv:1109.3472].

[17] J. P. Ader, J. M. Richard and P. Taxil, Phys. Rev. D 25, 2370 (1982).

[18] A. V. Manohar and M. B. Wise, Nucl. Phys. B 399, 17 (1993) [hep-ph/9212236].

[19] B. A. Gelman, S. Nussinov, Phys. Lett. B 551, 296 (2003) [hep-ph/0209095].

[20] Y. Frishman and M. Karliner, JHEP 1308, 096 (2013) [arXiv:1305.6457 [hep-ph]].

[21] T. Aaltonen et al. [CDF Collaboration], Phys. Rev. Lett. 100, 182002 (2008) [arXiv:0712.1506 [hep-ex]].

[22] M. Karliner and J. L. Rosner, Phys. Rev. D 90, no. 9, 094007 (2014) [arXiv:1408.5877 [hep-ph]].

[23] T. Aaltonen et al. (CDF Collaboration), Phys. Rev. Lett. 100, 182002 (2008) [arXiv:0712.1506 [hep-ex]]. 
[24] V. M. Abazov et al. (D0 Collaboration), Phys. Rev. Lett. 101, 012001 (2008) [arXiv:0802.4258 [hep-ex]].

[25] R. Aaij et al. (LHCb Collaboration), Phys. Rev. Lett. 109, 232001 (2012) [arXiv:1209.5634 [hep-ex]]; arXiv:1408.0971 [hep-ex]; arXiv:1407.2126 [hep-ex]; Eur. Phys. J. C 74, 2839 (2014) [arXiv:1401.6932 [hep-ex]]; Phys. Rev. Lett. 111, 181801 (2013) [arXiv:1308.4544 [hep-ex]]; J. High Energy Phys. 09 (2013) 075 [arXiv:1306.6723 [hep-ex]]. Phys. Rev. D 87, 112012 (2013) [arXiv:1304.4530 [hep-ex]].

[26] K. Anikeev, D. Atwood, F. Azfar, S. Bailey, C. W. Bauer, W. Bell, G. Bodwin and E. Braaten et $a l$. " " $B$ physics at the Tevatron: Run II and beyond," Workshop on B Physics at Conferences C99-09-23.2 and C00-02-24, hep-ph/0201071.

[27] V. V. Kiselev and A. K. Likhoded, Phys. Usp. 45, 455 (2002) [Usp. Fiz. Nauk 172, 497 (2002)] [hep$\mathrm{ph} / 0103169]$.

[28] J. D. Bjorken, Fermilab preprint, "Estimates of Decay Branching Ratios for Hadrons Containing Charm and Bottom Quarks," 1986, FERMILAB-PUB-86-189-T, http://lss . fnal . gov/archive/1986/pub/fermilab-pub-86-189-t.pdf

[29] M. A. Moinester, Z. Phys. A 355, 349 (1996) [hep$\mathrm{ph} / 9506405]$.

[30] M. Karliner and H.J. Lipkin,hep-ph/0307243, Phys. Lett. B575 (2003) 249.
[31] M. Karliner, B. Keren-Zur, H. J. Lipkin and J. L. Rosner, arXiv:0706.2163v1 [hep-ph].

[32] M. Karliner, B. Keren-Zur, H. J. Lipkin and J. L. Rosner, arXiv:0708.4027 [hep-ph] (unpublished) and arXiv:0804.1575 [hep-ph], Annals Phys 324,2 (2009).

[33] N. Brambilla et al. (Quarkonium Working Group Collaboration), hep-ph/0412158.

[34] C. H. Chang and X. G. Wu, Eur. Phys. J. C 38, 267 (2004) [hep-ph/0309121].

[35] Y. N. Gao, J. He, P. Robbe, M. H. Schune and Z. W. Yang, Chin. Phys. Lett. 27, 061302 (2010).

[36] Y. Amhis et al. (Heavy Flavor Averaging Group Collaboration), arXiv:1207.1158v2 [hep-ex].

[37] R. Aaij et al. (LHCb Collaboration), Phys. Rev. D 85, 032008 (2012) [arXiv:1111.2357 [hep-ex]].

[38] R. Aaij et al. (LHCb Collaboration), J. High Energy Phys. 04 (2013) 001 [arXiv:1301.5286 [hep-ex]].

[39] LHCb Collaboration, LHCb-CONF-2013-011, presented at EPS Conference, Stockholm, 2013.

[40] R. Aaij et al. (LHCb Collaboration), arXiv:1405.6842 [hep-ex].

[41] Y. Kato et al. (Belle Collaboration), Phys. Rev. D 89, 052003 (2014) [arXiv:1312.1026 [hep-ex]].

[42] V. V. Kiselev, A. K. Likhoded and M. V. Shevlyagin, Phys. Lett. B 332, 411 (1994) [hep-ph/9408407].

[43] J. P. Ma and Z. G. Si, Phys. Lett. B 568, 135 (2003) [hep-ph/0305079]. 